\title{
Die Pariser Weltausstellung in deutschen Kulturzeitschriften
}

\author{
ALKE BROCKMEIER
}

Der Exposition internationale universelle de Paris 1900 wird unter allen anderen Weltausstellungen eine Sonderstellung zugeschrieben. Sie bildet nicht nur den Angelpunkt zwischen dem 19. und dem 20. Jahrhundert - eine Retrospektive und eine Vorausschau zugleich -, sondern sie gehört darüber hinaus auch mit ihrer Größe und ihrem Expositions-Spektrum zu einem der Höhepunkte in der Geschichte der Weltausstellung. Seit der ersten internationalen Ausstellung 1851 in London waren die Besucherzahlen stetig gestiegen - 1900 sind es mit 48 Millionen so viele wie nie zuvor. Bereits viermal war in Paris eine Weltausstellung ausgerichtet worden: 1855, 1867, 1878 und 1889. Diese Ausstellung nun umfaßt allein schon eine Fläche, die derjenigen aller vier zusammen entspricht. Bleibende Bauten der Ausstellung sind der Pont Alexandre III sowie der Grand und der Petit Palais. ${ }^{1}$ In einer Zeit wirtschaftlich-technischen Fortschritts und kulturell-politischen Umbruchs gewinnt die Weltausstellung an Bedeutung und an öffentlichem Interesse.

Die Zeitschriftenlandschaft um 1900 ist enorm einflußreich: Niemals wieder wurde eine so große Anzahl an programmatisch verschiedenartigen und inhaltlich vielschichtigen Zeitschriften publiziert, die die Wahrnehmung und Meinung ihrer Leser maßgeblich bestimmen. Wie unterschiedlich die Reaktionen auf die Weltausstellung dabei ausfallen, zeigt sich bei einem Vergleich unterschiedlicher Zeitschriftentypen. Um die Jahrhundertwende spielen in Deutschland Zeitschriften mit literarischem Schwerpunkt respektive reine Literaturzeitschriften eine immens große Rolle, wie beispielsweise die Gesellschaft, die Blätter für die Kunst, die Insel oder der Pan. In diesem Zeitschriftentypus herrscht Stillschweigen über das Pariser Großereignis. Nicht nur deutsche Schriftsteller ignorieren diese Weltausstellung schlichtweg, sondern auch in Frankreich selbst findet sie unter Autoren wenig Zustimmung und Interesse. Ein halbes Jahrhundert zuvor war das noch ganz anders: Die Pariser Weltausstellung von 1867 wurde von einem Paris Guide par les principaux écrivains et artistes de la France begleitet, für den Taine, Dumas fils, Gautier, Renan, SainteBeuve und allen voran Hugo Beiträge geschrieben hatten. Mit der Zeit entwi-

1 Darstellungen der Pariser Weltausstellung von 1900 findet man bei Gaillard, S. 74-142, Krutisch, S. 95-103, sowie bei Mandell und Ory. 
ckelte sich jedoch die Weltausstellung ausgehend von einem instruktiven Wissensaustausch hin $\mathrm{zu}$ einem unterhaltenden Massenereignis, was mit einer wachsenden Ausstellungsmüdigkeit bei Teilen der Gesellschaft einherging (vgl. Wörner, S. 5). In Flauberts Dictionnaire des idées reçues findet sich unter dem Eintrag EXPOSITION die Definition: „Sujet de délire du XIX ${ }^{\text {ème }}$ siècle“. Octave Mirbeau schreibt in der Revue des deux mondes: „J'ai beau chercher, je ne trouve pour la justifier rien d'autre que cette superstition populaire, que les expositions universelles doivent revenir chez nous, tous les dix ans, comme les grandes gelées“ (Band 132, S. 888). Und Zola, der anläßlich der Weltausstellung von 1900 um einen Beitrag für die Grande Revue gebeten worden war, verweigert jede Äußerung zu diesem Thema: ,Je ne suis pas de cette Exposition, et je ne veux pas en être, par aucune manifestation." ${ }^{2}$

Andere, nicht schwerpunktmäßig literarische Zeitschriftentypen schenken der Weltausstellung um so mehr Aufmerksamkeit, so auch derjenige der ,Rundschau'. Geprägt von der Deutschen Rundschau, die Julius Rodenberg nach den Vorbildern der Revue des deux mondes und der Quarterly Revue gründet, gewinnt sie in den letzten Jahrzehnten des 19. Jahrhunderts durch die Neue Deutsche Rundschau (der vorherigen Freien Bühne) und Nord und Süd an Bedeutung. Das erklärtermaßen politische Profil der Deutschen Rundschau ist zugleich mit dem Anspruch verbunden, die „Gesammtheit der deutschen Culturbestrebungen" aufzugreifen. ${ }^{3}$ Sie will zugleich unterhalten und eine breite Spannweite an Ressorts und Themen wissenschaftlich präsentieren.

Erfolgreich und auflagenstark war außerdem der Typus der ,Familienzeitschrift' (oder ,Illustrierten Monatsschrift'), die zum Genre der Unterhaltungszeitschriften gezählt wird. ${ }^{4}$ Gattungsprägend für die Unterhaltungszeitschrift ist vor allem, daß sie aufwendig illustriert ist. Dem liegt ein Ansatz zugrunde, den die Illustrierte Zeitung bereits 1868 programmatisch formuliert: Der Einsatz von Illustrationen soll der ästhetischen und instruktiven Volksbildung dienen und „was Wissenschaft und Kunst früher nur der Minorität bevorzugter Geister darzubieten vermochte, im edelsten Sinne des Wortes [...] popularisieren" (Band 51, S. 4). So heißt es auch im Vorwort der ersten Ausgabe von Westermanns illustrierten deutschen Monatsheften, dem „Mangel eines größeren Centralorgans für die nach Volksthümlichkeit ringende Bildung unserer Zeit" Abhilfe leisten zu wollen. Der Weltausstellung wird in diesem Zeitschriftentypus große Beachtung geschenkt. Velhagen \& Klasings Monatshefte führen im Weltausstellungsjahr sogar eine eigene Rubrik unter dem Titel Von der Weltausstellung zu

2 Zit. nach Mitterand, S. 703. Zola lehnt nicht ohne Überlegung ab. Seine Distanzierung von der Weltausstellung begründet er damit, daß diese darauf ausgerichtet sei ,à chanter glorieusement notre fin de siècle“. Dem will er sich zwar nicht anschließen, was ihn aber nicht daran hindert, die Weltausstellung zu besuchen und fotografisch festzuhalten.

3 Gründungsprospekt Deutsche Rundschau, zit. nach Butzer/Günter, S. 116.

4 Einen umfassenden Überblick über Familien- und Unterhaltungszeitschriften mit ausführlicher Bibliographie bietet Graf. 
Paris, deren ausführliche und reich bebilderte Beiträge in den Monaten Juni, Juli und August erscheinen und mit der Zeit an immer prominentere Position rücken. So wird das Augustheft schließlich mit der Weltausstellungsrubrik eröffnet.

Noch umfangreicher, wenn auch weit weniger informativ, sind die als erzählende Erfahrungsberichte angelegten „Spaziergänge durch die Weltausstellung in Paris" des Familienblattes Die Gartenlaube. ${ }^{5}$ Das Familienblatt ist konzeptionell äußert leserorientiert: Mann, Frau, Kind - jedes Familienmitglied soll angesprochen werden. In den Weltausstellungsbeiträgen der Gartenlaube wiederholt sich dabei in ausdrücklicher und auffalliger Weise die Anrede der „Leserin“ und Einwürfe wie „Doch meine Leserinnen seufzen schon: Immer nur Maschinen!“ (Band 48, S. 703). Tatsächlich galt das Blatt, spätestens seit Abdrucken von Romanen der Marlitt, als ausgesprochene Frauen-Zeitschrift (vgl. Kirschstein, S. 101 f.). Die „Spaziergänge durch die Weltausstellung“ tragen dem auch durch ihre Themenwahl Rechnung: so wird der Leserin die Textilienausstellung empfohlen und generell zwischen „Damen- und Herrenausstellung" unterschieden (Band 48, S. 701).

Zunächst stellt sich die Frage danach, welche Bereiche der Weltausstellung in Rundschauen und Familienzeitschriften vertreten sind und in welcher Gewichtung sich die Beiträge mit ihnen befassen. Augenscheinlich wurde das Phänomen Weltausstellung - wie auch heute noch - aus sehr unterschiedlichen Perspektiven und mit nicht weniger verschiedenen Wertungen betrachtet. Neben der unzweifelhaften politischen und wirtschaftlichen Bedeutung von Weltausstellungen steht dabei diejenige von Kultur und Unterhaltung; Teilaspekte, unter denen sich die zeitgenössische Berichterstattung differenzieren läßt. Entsprechend ihrer Programmatik ist es hier die Deutsche Rundschau, die auch in ihrer Rubrik Politische Rundschau regelmäßig von der Weltausstellung berichtet.

Sowohl der Typus der Rundschau als auch die Familienzeitschrift Westermanns Monatshefte berücksichtigen in besonderer Weise das Thema „Kunst auf der Pariser Weltausstellung“. Unter diesem Titel erscheint eine Besprechung in der Deutschen Rundschau, deren Autor Walther Gensel auch an einem der groBen Kataloge mitgearbeitet hatte: Die Pariser Weltausstellung in Wort und Bild, herausgegeben von Georg Malkowsky (Berlin 1900). Von Beginn an hatte die Zeitschrift einen kulturgeschichtlichen Anteil und bereits seit 1880 eine eigene Rubrik Kunst und Kunstgeschichte (vgl. Syndram, S. 351). Dementsprechend reiht sich auch der Beitrag über die Kunst auf der Weltausstellung in eine lange

5 Ernst Keil gründet 1853, ein Jahr nach dem erstmaligen Erscheinen der Unterhaltungen am häuslichen Herd bei Brockhaus, mit der Gartenlaube das erfolgreichste Familienblatt. Velhagen \& Klasing reagieren darauf, indem sie neben den Monatsheften eine weitere illustrierte Zeitschrift, den Familienblatttypus Daheim publizieren. 
Reihe von Artikeln über nationale Kunstausstellungen ein. ${ }^{6}$ Die Neue Deutsche Rundschau widmet sich mit einem umfangreichen kunstwissenschaftlichen Abriß der französischen Centennarausstellung (Band 11, S. 1284-1304), der zugleich ein Vorabdruck einer größeren Buchpublikation des österreichischen Kunsthistorikers Richard Muthner ist. Ein Jahrhundert französischer Malerei erscheint 1901, ebenfalls im Fischer-Verlag. In den Familienzeitschriften dagegen wird das Kunstressort nahezu vollständig ausgespart. Für Velhagen $\mathcal{E}$ Klasings Monatshefte sind nur die deutschen Kunst- und Kunstgewerbeausstellungen einer vergleichsweise knappen Erwähnung wert (Band 14.1, S. 520-522), was ebenso für Die Gartenlaube gilt. Die stilistisch blumigen Ausführungen der Gartenlaube beziehen sich außerdem auf Objekte, die wohl eher dem Kunst-Kitsch zuzurechnen sind. Die deutsche Porzellanmanufaktur löst die Begeisterung des Berichtenden aus, namentlich ein ,märchenschöne[s] Stück, auf dem ein zartumrissener Engelskopf aus einem krystallenen Schneeflockenreigen schaut", wird als „Schöpfung[ edelster Kunst“ herausgestellt (Band 48, S. 546).

Eine Ausnahme unter den Familienzeitschriften stellt daher der Kunstbeitrag in Westermanns Monatsheften dar (Band 89, S. 668-685). Die Zeitschrift hatte in den 80er Jahren des 19. Jahrhunderts unter dem Konkurrenzdruck anderer illustrierter Periodika ihren konzeptionellen Schwerpunkt auf die bildenden Künste gelegt und beschäftigte zahlreiche Kunsthistoriker, darunter Max Jordan, Eugen Kalkschmidt und Hans Rosenhagen. Der Autor des notabene einzigen Weltausstellungsartikels in Westermanns Monatsheften berichtet ausschließlich über die dekorative Kunst und damit über einen Bereich, der mit der Bewegung des Art Nouveau zum besonderen Erfolg der Pariser Ausstellung beiträgt. Die neue Kunstrichtung ist die große, ins 20. Jahrhundert führende Neuheit und Sensation und bestimmt nicht nur die eigentlichen Ausstellungen wie eben diejenigen der so genannten , dekorativen Kunst'. Sie prägt auch die Gestaltung von Katalogen ${ }^{7}$ und vor allem in kleinen Details die Architektur der Stadt; so etwa den berühmten von Hector Guimard gestalteten Eingangsbereich der ersten Metrolinie, die im Juni 1900 anläßlich der Weltausstellung eingeweiht wird. Sie fährt zwischen dem zentralen Hauptgelände der Ausstellung und dem ausgelagerten Bereich in Vincennes, in dem Eisenbahnen und Automobile ausgestellt sind und auch die Olympischen Spiele stattfinden.

Die wirtschaftliche Seite von Weltausstellungen trifft bei vielen Besuchern und Beobachtern - auch aus der Retrospektive - auf Kritik. Walter Benjamins

6 In den Jahren um 1900 sind das in chronologischer Reihenfolge: Julius Lessing: „Das Kunstgewerbe auf der Berliner Kunstausstellung.“ In: Deutsche Rundschau 81 (1894) 230-234; Walther Gensel: „Berliner Kunstausstellungen.“ In: Deutsche Rundschau 108 (1901) 300-307 und ders.: „Die Kunst auf der Düsseldorfer Ausstellung.“ In: Deutsche Rundschau 112 (1902) 294-302.

7 So auch den offiziellen deutschen Katalog, der von dem Künstler Bernhard Pankok graphisch im Jugendstil gestaltet worden war. 
in diesem Zusammenhang oft zitierter Ausspruch „Weltausstellungen sind Wallfahrtstätten zum Fetisch Ware"8 steht sinnfällig dafür, daß sich 1900 eine regelrechte Tourismusindustrie entwickelt: „Man konnte in Berlin, München, Frankfurt oder Stuttgart Schlafwagen, Hotel und verschiedene Ausflüge als komplettes Angebot buchen" (Kuchenbuch, S. 137). Eben diesem kommerziell-touristischen Charakter der Weltausstellung tragen Westermanns Monatshefte Rechnung, indem sie den Reisenden sowohl Paris-Führer (Band 88, S. 836 f.) als auch Ausstellungskataloge wie Die Kunstsammlung Friedrich des Großen auf der Pariser Weltausstellung empfehlen (Band 89, S. 147 f.).

Die Weltausstellung als solche war zudem immer dem Vorwurf ausgesetzt, lediglich ,eine destillierte Scheinwelt“ zu errichten (Hofmann, S. 152). Diese sei, so Kritiker, Dokument und Ausdruck „der europäischen Glaubenskrise“. Die „vorhandene Wirklichkeit“ werde dabei „durch ihren künstlichen Ersatz entwertet und verdrängt", was sich in krasser Form im Vieux Paris der Weltausstellung von 1900 zeige (Ebd., S. 168 und 177). Der Nachbau einer mittelalterlichen Straße am Ufer der Seine rekonstruiert nicht nur verschwundene Gebäude, sondern eben auch solche, die real in nur geringer Entfernung besichtigt werden könnten. Weniger harsch urteilt die Neue Deutsche Rundschau über die illusionistische Seite der Ausstellung. Sie faßt die Ausstellung ebenfalls als ausdrücklich künstliche Welt auf, erinnert aber wiederholt an Autoren, Figuren und Motive französischer Décadence-Literatur wie den Protagonisten des Romans À Rebours, des Esseintes. Nicht nur der Besucher müsse diese Verbindung herstellen, auch die Aussteller selbst täten dies. Über die Schmückkünstler des Art Nouveau wie Lalique und Gallé heißt es:

Sie haben alle einen litterarischen Zug. Sie haben Baudelaire gelesen und Poe, sie wissen sich Flaubert verwandt und sie wünschen sich Nervenkünstler wie Huysmans' Des Esseintes zu Verstehern und Erwerbern. Sie wollen mit ihren Schmuckstücken innere Vorstellungswelten zum Ausdruck bringen und sie feilen und fügen ihren Stoff mit der Sorge der Parnassiens. (Band 11, S. 888)

Als 1851 in England der Gedanke einer Weltausstellung zum ersten Mal verwirklicht wird, heißt es in der Eröffnungsrede, Ziel des Unterfangens sei die „Verwirklichung einer Einigung der Menschheit“ (Velhagen \& Klasings Monatshefte, Band 14.1, S. 50) - ein Motto, das auch den nachfolgenden Ausstellungen gelten sollte. Ein halbes Jahrhundert später jedoch steht die Weltausstellung vor allem unter nationalistischen Vorzeichen. Über die Eröffnungsreden der Ausstellung von 1900 heißt es denn auch in Velhagen E Klasings Monatsheften: „Beiläufig: es war in diesen Reden so viel vom lieben Frieden die Rede, daß einem ordentlich bange werden konnte" (Band 14.2, S. 378). Anläßlich der Jahrhundertwende wird im Rückblick vielfach die nationale Einheit beschworen, so auch in der Neuen Deutschen Rundschau - bezeich-

8 Benjamin, S. 412. Marx hatte im Ersten Buch des Kapitals der Ware Fetischcharakter zugeschrieben. 
nenderweise auf der ersten Seite der ersten Ausgabe des Jahres 1900: „Was uns Deutschen vor hundert Jahren gefehlt hat, ist klar: Der Staat und das staatliche Bewußtsein, der Zusammenschluß zu Einem Volk und die nationale Gesinnung, Vaterland und Vaterlandsliebe." (Band 11, S. 1) Wehler beschreibt die „Radikalisierung des Reichsnationalismus“ der Zeit unter anderem als „Naturalisierung des Nationsbegriffs durch die moderne, von Darwin inspirierte Biologie und dem populärwissenschaftlich verkürzten Sozialdarwinismus“" (Wehler, S. 80). Ein Konzept, das als „l'universelle lutte pour la vie“ nicht nur auf französischer Seite ausdrücklich mit dem nationalen Kräftemessen der Pariser Weltausstellung in Verbindung gebracht wird. ${ }^{9}$

Frankreich und Deutschland stehen in besonderer Rivalität zueinander, was sich bereits direkt im Anschluß an die Weltausstellung von 1889 äußert, als beide Länder die Ausrichtung der kommenden Ausstellung ins Auge fassen. Ohnehin ist der Krieg von 1870/71 unvergessen und bildet den Nährboden für die anhaltende deutsch-französische Konkurrenz, während gerade in der Weltausstellung von 1900 nicht von ungefähr nicht nur die Folge dieses Krieges, sondern auch eine „guerre mondiale symbolique“ (Prochasson, S. 99) und damit die sinnbildliche Vorwegnahme des Ersten Weltkrieges gesehen wird: Der Palais des ministères de la guerre et de la marine bietet dabei das Anschauungsmaterial nationalen Wettrüstens und Zähnezeigens. Deutschland hatte außerdem weder 1878 noch 1889 an der Weltausstellung in Paris teilgenommen. Die Entscheidung von 1878 war eine allein deutsche und ging auf Bismarck zurück, der als Begründung äußerte:

Wenn wir dieser durch die unzweideutigsten Tatsachen kundgegebenen, nur Нaß und Verachtung kennzeichnenden Stimmung der Franzosen gegenüber eine an alle Welt ergangene Einladung derselben gewissermaßen als Gäste Frankreichs annehmen, so würden wir damit meines Erachtens einen bedauerlichen Mangel an nationalem Ehrgefühl bekunden. (Zit. nach Haltern, S. 8)

Dahingegen hatte das hundertjährige Jubiläum der Französischen Revolution neben dem Deutschen Reich auch andere Monarchien dazu veranlaßt, die Weltausstellung in Paris zu boykottieren: Österreich-Ungarn, Russland und auch Großbritannien. ${ }^{10}$ Frankreich sichert sich schon 1892 vor Deutschland die prestigeträchtige Jahrhundertwende-Ausstellung; statt der Weltausstellung findet in Berlin 1896 eine (von Frankreich belächelte) Industrieausstellung statt (vgl. Prochasson, S. 96 f.). Nur selten findet dieser Sachverhalt in der deutschen Presse Erwähnung: Velhagen \& Klasings Monatshefte berichten zwar von deutschen Überlegungen, zur Jahrhundertwende eine Weltausstellung ausrichten $\mathrm{zu}$ wollen, jedoch habe es - und das ist in betont gleichgültigem und scheinbar überlegenem Ton formuliert - die „Bismarcksche Politik [...] stets

9 Eine Formulierung von Jules Roches, zit. nach Prochasson, S. 95. Auch Naumann bedient sich in den Pariser Briefen sozialdarwinistischen Vokabulars (vgl. Naumann, S. 93).

10 Velhagen \& Klasings Monatshefte stellen dies mit Nachdruck heraus (Band 14.1, S. 55). 
vermieden [...], nach französischem Muster für das Prestige zu arbeiten" (Band 14.1, S. 56).

Derselbe Beitrag verurteilt denn auch die Pariser Weltausstellung von 1878, die nach der kriegsbedingten Schwächung Frankreichs noch gar keine nennenswerte Ausstellung habe sein können, und bespöttelt das Wahrzeichen der Weltausstellung von 1889, den Eiffelturm, der auch im Jahr 1900 in das Ausstellungskonzept eingegliedert ist: Er habe „,weit über Gebühr Aufsehen erregt" (Ebd.). Die Porte monumentale bildet den Haupteingang der Ausstellung von 1900 und ist einer ihrer symbolträchtigen Bauten. In der Revue de Paris wird das Tor folgendermaßen beschrieben: „imposante comme une cathédrale, colorée comme une mosquée et ciselée comme une pagode". ${ }^{11}$ Velhagen $\mathcal{E}$ Klasings Monatshefte dagegen, die eine fotografische Abbildung der Porte monumentale drucken (Band 14.2, S. 379), überziehen sie mit Polemik:

Wunderlich: die größte aller Geschmacklosigkeiten, welche die Ausstellung aufzuweisen hat, steht an ihrem Anfang. Mich überlief geradezu ein Gruseln, als ich zum ersten Male vor dem Haupteingangsthor am Place de la Concorde vorfuhr. Man denke sich eine ungeheure, ganz in Bayerisch-blau angepinselte, an den Seiten durchbrochene Käseglocke; rechts und links derselben je einen bunten Riesenspargel, dann hat man die beste Vorstellung dieses scheußlichen Machwerks. Aber damit noch nicht genug: besagte Käseglocke hat vorn einen krankhaften Auswuchs, und auf diesem thront die Gestalt einer ganz modernen Pariserin, welche gleichsam die Gäste willkommen heißen soll... man nennt sie hier bereits kurzweg „la poupée“ und trifft damit den Nagel auf den Kopf. Sie ist so fad, so gesucht, so abscheulich, wie das ganze Riesenthor. (Band 14.2, S. 380)

So sehr einerseits das Eingangstor kritisiert wird, so großes Lob lassen andererseits vor allem die Familienzeitschriften dem Deutschen Haus zuteil werden. Auch diesem Bau wird symbolische Bedeutung zugeschrieben; - unter den 22 Pavillons der Rue des Nations heben ihn Velhagen \& Klasings Monatshefte folgendermaßen hervor: „Weithin leuchtend überragt auf 75 Meter Höhe die Turmspitze des deutschen Hauses alle übrigen Paläste der Rue des Nations. Immer wieder kehrt das Auge zu dem frischen Bau zurück, der sich jedenfalls aus der ganzen Reihe kräftig und eigenartig heraushebt." (Band 14.2, S. 516) Die Gartenlaube widmet den „Deutschen Bauten auf der Pariser Weltausstellung“ sogar einen ganzen, selbstverständlich bebilderten Artikel. Hier lautet der erste Satz: „Unter den als Gäste erschienenen Nationen, die sich auf der französischen Weltausstellung durch eigene Gebäude hervorgethan haben, zählt Deutschland zu den türmereichsten" (Band 48, S. 352). Wo also keine Besonderheit deutscher Beteiligung besteht, wird schlicht die Anzahl der Türme zur Wettkampfkategorie erklärt. Diese Tendenz, die architektonischen Schöpfungen anderer Länder mangelhaft und zugleich diejenigen Deutschlands übertrieben positiv zu bewerten, läßt sich wohlgemerkt nicht nur in den untersuchten

11 Michel Corday (d. i. Louis-Léonard Pollet): „La Genèse de l'Exposition.“ In: Revue de Paris, Bd. 132, 15. Juli 1899, S. 430. Zit. nach Prochasson, S. 100. 
Zeitschriften feststellen. Auch Friedrich Naumann, der in seinen Pariser Briefen von der Weltausstellung berichtet, mokiert sich über das Eingangstor: „Es will originell sein und kann es nicht" (Naumann, S. 74). Und auch er streicht heraus, daß das deutsche Haus die umliegenden Bauten überragt; sein lobendes Urteil über die Architektur des Hauses ist auffallend pauschal: „Die Stilart des deutschen Pavillons ist im Vergleich zu ihrer Umgebung immer gut und nobel." (Ebd., S. 79 f. und 77)

Doch vor dem Hintergrund, die konkurrierenden Länder in möglichst vielen Bereichen übertrumpfen zu wollen, gab es auf deutscher Seite eine große Enttäuschung: ,[...] man hatte doch wohl ein Recht darauf, den größten aller deutschen Industriellen hier auf dem Platze zu erwarten...", heißt es in Velhagen \& Klasings Monatsheften (Band 14.2, S. 520). Einhellig wird die Abwesenheit Krupps beklagt, der auf der Londoner Weltausstellung mit seinem Gussstahlblock und der Stahlkanone beeindruckt hatte und auf den folgenden Ausstellungen als ,Kanonenkönig' bewundert wurde. Westermanns Monatshefte hatten ein Jahr zuvor, wohl auch in Hinblick auf die Weltausstellung, einen langen biographischen Text zu Ehren Alfred Krupps abgedruckt (Band 86, S. 69-91). Krupps Kanone war im neunzehnten Jahrhundert an viele Länder verkauft worden - jedoch nicht an Frankreich. ${ }^{12}$ Wolbring ruft in Erinnerung, daß die Kruppsche Riesenkanone aus der Perspektive Frankreichs eng an den Krieg und die Niederlage von 1870/71 gebunden ist, ,als der martialische deutsche Militarismus über die friedfertige, im Grunde höherstehende französische Kultur habe siegen können “. ${ }^{13}$ Auch Krupp selbst verkündet den Symbolcharakter seiner Stahlkanonen im Krieg gegen Frankreich: „Wir leben jetzt in der Stahlzeit. Das Eisenbahnwesen, Deutschlands Größe, Frankreichs Sturz, fällt in die Stahlzeit. Die Bronzezeit ist dahin “. ${ }^{14}$ Neben der rein technikbezogenen Konkurrenz der Weltausstellung beschwört die Neue Deutsche Rundschau also auch den militärischen Mythos Krupp, wenn sie schreibt: „Und wenn auch Deutschland als maitre des Forges seine Räder- und Hammerwerke brüllend erdröhnen läßt, ein Faktor würde der Bilanz, die man in diesen imposanten Hallen noch am sichersten ziehen könnte, doch auch fehlen: die Kanonen Krupps." (Band 11, S. 884)

Zur generellen patriotisch-nationalistischen Färbung der Weltausstellung von 1900 trägt die Kolonialausstellung bei, die zum ersten Mal als eigene Ausstellungskategorie geführt wird. Sie umfaßt zur Hälfte französische Kolonien; den übrigen Ausstellungsplatz am Trocadéro teilen sich Großbritannien, die Niederlande, Portugal, Sibirien und Russland, Ägypten, China und Japan. Ob ein Land in die Kolonialausstellung aufgenommen wurde, entschied sich nicht

12 Vgl. dazu Wolbring, besonders das Kapitel Krupps Triumphe auf den Weltausstellungen, S. 85-122.

13 Ebd., S. 108. Wolbring bezieht sich auf E. Malos „L'Art de tuer“. Guide de l'Exposition universelle de 1889.

14 Alfred Krupp an Wilhelm I., 23. April 1871, zit. nach Gall, S. 161. 
danach, ob es den kolonialen Kriterien entsprach, sondern nach seiner (subjektiv eingestuften) Exotik. Die Familienzeitschriften sind es, die die koloniale Ausstellung explizit erwähnen und kommentieren. Die Gartenlaube glorifiziert dabei das imperialistische Zeitalter: ,,[...] die Kolonialausstellungen lehren uns, wie die Erde für die Menschheit größer geworden ist, und geben uns das weltgeschichtliche Schauspiel ihrer Aufteilung an die Kultur der vorgeschrittenen Nationen." (Band 48, S. 770) Der Kolonialausstellung von 1900 wird heute mit ihrem realitätsfernen und unterhaltenden Charakter ein großer Einfluß auf die allgemeine Einstellung der Bevölkerung gegenüber Kolonialisierung zugeschrieben, ein Einfluß mit historischer Wirkung (vgl. dazu Schneider). Für Velhagen \& Klasings Monatshefte bietet sie eine Gelegenheit, erneut polemisch auf das Gastgeberland zu zielen, das seinem ,alten und neuen Kolonialbesitz ein Denkmal neben dem anderen“ errichtet habe, ,um den lieben Landsleuten und der Welt gründlich zu zeigen, wie herrlich weit man es gebracht hat [...]!“” (Band 14.2, S. 582)

In der Konkurrenzsituation werden auch die nationalen Stereotype ,männ-

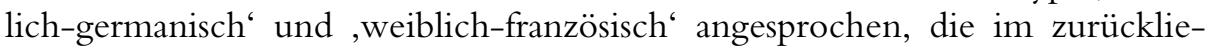
genden Krieg gegen Frankreich gestärkt worden waren. ${ }^{15}$ Zola hatte in La Débâcle konstatiert, daß Frankreich in diesem Krieg „la virilité de se battre“ fehle (Zola, S. 524). Auf deutscher Seite konstruiert sich die Gleichsetzung von ,männlich' und ,kriegerisch' als feststehende Eigenschaft des deutschen ,Nationalcharakters', deren Beschwörung später auch die Propaganda des Ersten Weltkriegs bestimmen sollte. Wie der Nationalismus selbst müssen, so Wehler, auch begleitende Vorstellungen von „Völkerpsychologie“ und „Nationalcharakter" unter den Mechanismen des Konstruktivismus betrachtet werden (Wehler, S. 7-9). Auch Florack definiert das nationale „Stereotyp“ als sozialpsychologischen Begriff, der auf subjektive „Wahrnehmungsmuster" zurückgehe (Florack, S. 12). Die Porträtmalerei der Weltausstellung wird in der Deutschen Rundschau - unter polarisierendem Rückgriff auf die genannten Stereotype wie folgt beschrieben: „Bei den männlichen Porträts stehen die germanischen Völker entschieden an erster Stelle. [...] Bei den Frauenbildnissen treten natürlich die Franzosen am meisten hervor." (Band 104, S. 265) Hier ist exemplarisch zu beobachten, was Wehler als typischen Nationalisierungsprozeß beschreibt: „Kunstwerke wurden als Ausdruck des Nationalcharakters interpretiert." (Wehler, S. 49) Aller Wettbewerb wird auf den Gastgeber fokussiert, dessen vermeintliche oder simplifiziert dargestellte Stärken und Schwächen das Bild von einem Frankreich ergeben, das sich auf zweifelhaften Gebieten als dominierend erweist, wesentliche Tugenden aber vermissen läßt:

Bei den eigentlichen Luxusgegenständen zeigt sich [Frankreich] noch vollkommen überlegen; wo es aber gilt, den Geschmack fürs Tüchtige und Gute auch in weitere Kreise zu tragen, sind die anderen Völker mindestens ebenbürtig. [...] Die

$15 \mathrm{Daß}$ dieser Geschlechterbezug deutsch-französischer Stereotype eine lange Tradition hat, zeigt Florack, S. 319-338. 
größten Anstrengungen hat jedenfalls Deutschland gemacht, und so schneidet es denn auch hier ganz besonders günstig ab. (Deutsche Rundschau, Band 104, S. 445)

In diesem Sinne häufen sich Varianten deutsch-französischer bzw. nordsüdlicher Stereotype. Derselbe Autor erklärt volkstümelnd den Norden zur „eigentliche[n] Heimath des Intérieurs“, denn:

Je weiter man nach Süden kommt, desto mehr leben die Menschen im Freien, desto weniger kennen sie den Comfort, die Traulichkeit der Zimmer. Nur im Norden versteht man die Poesie der Abendlampe, des brodelnden Theekessels, konnte die Sitte des Christbaumes sich einbürgern und erhalten. (Deutsche Rundschau, Band 104, S. 267)

Selbst die Zusammenstellung von Ausstellungsgegenständen und das Verhalten der Besucher werden, wie in der Gartenlaube, vor dem Hintergrund behaupteter nationaler Eigenschaften kommentiert. So sei die französische Kunstgewerbeausstellung „ein einziger großer Bazar“, die Kunstgewerbeausstellung anderer Nationen dagegen

[...] zeichnet sich vor der französischen Abteilung, die doch so viel Schönes enthält, durch eine gewisse vornehme Ruhe aus. Das Vielerlei zum Teil alltäglicher Dinge, dem wir in der französischen Ausstellung begegnen, weicht einer würdevollen Wahl der Schaugegenstände, und obwohl auch hier gekauft und verkauft wird, sind die Säle doch nicht durch ein lärmendes Treiben entweiht. (Band 48, S. 545)

Der Besucher der Weltausstellung prägt darüber hinaus auch die Illustrationen der Gartenlaube, die anders als diejenigen der Monatshefte Velhagen \& Klasings und Westermanns zumeist nicht fotografiert, sondern gezeichnet sind. Sie zeigen bemerkenswerte Charakteristika: Zunächst dominiert der Besucher immer, vor dem unscheinbar in den Hintergrund gerückten Ausstellungsobjekt, das Bild. Das korreliert im Übrigen mit den Texten der Gartenlaube, in die häufig Dialoge und Kommentare von Ausstellungsbesuchern eingestreut sind, die in auffälliger Weise stets die deutschen Beiträge loben. Die Illustration Im Weinrestaurant des deutschen Hauses: ein Hoch auf die Heimat (Band 48, S. 547; siehe Anhang, Illustration 1) begleitet die Sätze: „Der Rheinwein perlt, die Römer erklingen, das deutsche Lied wird lebendig, es rauscht in die von künstlichen Sonnen erhellte Nacht. Und im schwellenden Lied vergessen wir für eine Stunde, wie fern wir der Heimat sind!“ (S. 548) Die nationale Tendenz der auflagenstarken Gartenlaube wurde vielfach untersucht und zählt geradezu zu den Grund-Eigenschaften eines typischen Familienblattes (vgl. dazu Barth und Koch). Daß auch rassistische Ideologie zu ihren Merkmalen gehört, führt beispielsweise die Illustration In der Maschinenhalle vor Augen (Band 48, S. 642; siehe Anhang, Illustration 2). Die Zeichnung zeigt eine Ausstellungsbesucherin, die ihre Blicke nicht auf das Ausstellungsobjekt, sondern auf einen rassistisch typisierten farbigen Besucher richtet. Zum einen werden mit diesen Bildbeispielen über die eigentlichen Textbeiträge der Gartenlaube hinaus auch über 
die Illustrationen patriotisch-nationalistische und rassistische Inhalte transportiert, zum anderen evoziert gerade das letztgenannte Bild einen Aspekt der Kolonialausstellung, über den in den Beiträgen nicht geschrieben wird. Die so genannte ,Völkerschau' hatte sich seit Mitte des 19. Jahrhunderts als fester Bestandteil der Kolonialausstellungen etabliert. In Deutschland ist es vor allem Carl Hagenbeck, der die Exposition von Menschen aus Afrika und Übersee populär gemacht hatte. Auch im Jahr 1900 bestimmt die Vorführung von Menschen wesentlich das Konzept der Kolonialausstellung. Ganze Dörfer werden nachgebaut, ihre Bewohner nach Paris gebracht und zur Schau gestellt.

Trotz der Unterschiede, die in der ideologischen Ausprägung der Beiträge evident geworden sind, ist in Rundschauen und Familienzeitschriften der Tenor des abschließenden Fazits derselbe: „Das Bemerkenswertheste der Ausstellung von 1900 ist das starke Hervortreten der deutschen Arbeitsleistung“, heißt es beispielsweise in der Deutschen Rundschau (Band 105, S. 291). Die Bewertungen bemessen vor allem die Bedeutung der Weltausstellung für das deutsch-französische Verhältnis. Für die Deutsche Rundschau haben sich - dank der Ausstellung der Kunstsammlung Friedrich des Großen - beide Länder einander angenähert. Sie schreibt:

Insbesondere hat die Weltausstellung auch dazu beigetragen, die eine oder andere Spitze im Verkehr zwischen Deutschland und Frankreich abzuschleifen. Das Interesse, das Kaiser Wilhelm II. der Ausstellung bewies, indem er Meisterwerke der französischen Kunst des achtzehnten Jahrhunderts aus preußischen Schlössern in das deutsche Haus der rue des Nations sendete, ist auch in den maßgebenden französischen Kreisen im Hinblick auf die symbolische Bedeutung dieses friedlichen Actes in vollem Maße gewürdigt worden, und die öffentliche Meinung in Frankreich hat dies Urtheil rückhaltlos dadurch anerkannt, daß sie dem deutschen Haus auf der Weltausstellung besondere Aufmerksamkeit schenkte. (Band 105, S. 306)

Andere Zeitschriften suchen nicht die Versöhnung. Die Gartenlaube triumphiert: „das ist ein neues Sedan - das ist die Niederlage der französischen Industrie!" (Band 48, S. 546) Ein vergleichbarer Ausspruch findet sich auch in Berichten französischer Zeitzeugen, so etwa in Paul Morands 1900:

[...] les machines des autres pays n'étaient plus que des jouets. Les vieux messieurs se faisaient transporter en fauteuil roulant, parmi cet amoncellement de soupapes, de volants, de régulateurs, et disaient en hochant la tête: „Notre Exposition est un Sedan commercial.“ (Morand, S. 93)

Damit offenbart der eigentlich friedliche Wettkampf der Länder zugleich seine weltpolitische Dimension und Brisanz - Versöhnungswillen aber auch Machtstreben, Friedenshoffnungen aber auch Ängste und Ressentiments (vgl. dazu Mandell, S. 28). Das Phänomen Weltausstellung für sich genommen besitzt bereits ein großes Potential, nationale Identität zu stiften und die Entwicklung von Nationalismus zu befördern. Vor dem Hintergrund der von der neueren Nationalismusforschung aufgestellten These, Nationalismus entstehe als „Legitimations-, Integrations- und Mobilisierungsideologie“ in Reaktion 
auf Modernisierungskrisen (Wehler, S. 63), erscheint das Nebeneinander von hochmodernem technischen und wissenschaftlichen Fortschritt und volkstümelndem Konservatismus der Weltausstellung als nur vordergründiges Paradoxon. Deutsche Rundschauen und vor allem Familienzeitschriften stärken mit ihrer Darstellung der Pariser Weltausstellung von 1900 zeitgenössische nationale Stereotype und nationalistische Positionen.

\section{Anhang \\ Zeitschriftenbeiträge zur Weltausstellung}

\section{Deutsche Rundschau}

104 (1900) 257-274 und 436-453 - Walther Gensel: Die Kunst auf der Pariser Weltausstellung.

105 (1900) 290-295 - A. Schricker: Die Pariser Weltausstellung.

105 (1900) 305-309 - Politische Rundschau.

\section{Neue deutsche Rundschau}

11 (1900) 1-17 - Theobald Ziegler: Auf der Schwelle des neuen Jahrhunderts.

11 (1900) 878-891 - Felix Poppenberg: Folies universelles.

11 (1900) 1284-1304 - Richard Muther: Die ältere Generation der französischen Maler. Betrachtungen aus der Pariser Centennarausstellung.

\section{Westermanns illustrierte deutsche Monatshefte}

1 (1856) - Vorwort.

86 (1899) 69-91 - Herman Frobenius: Alfred Krupp. Eine Lebensskizze.

88 (1900) 836 f. - P. Dcke [sic]: Litterarische Rundschau [Empfehlung jüngst erschienener Paris-Führer].

89 (1900) 147 f. - F. D. [Friedrich Düsel]: Litterarische Rundschau [Empfehlung des Kataloges Die Kunstsammlung Friedrich des Großen auf der Pariser Weltausstellung].

89 (1901) 668-685 - W. Fred: Betrachtungen über dekorative Kunst auf der Pariser Weltausstellung.

\section{Velhagen \& Klasings Monatshefte}

14.1 (1899/1900) 49-56 - Charpentier: Weltausstellungen. Rückblick und Ausschau.

14.2 (1899/1900) 377-387 - Hanns von Zobeltiß: Von der Weltausstellung zu Paris. I. Der Rahmen. Mit vierzehn Abbildungen nach Aufnahmen von Lévy \& ses fils in Paris.

14.2 (1899/1900) 513-528 - Hanns von Zobeltiß: Von der Weltausstellung zu Paris. II. Die Rue des Nations. - Deutsche Kunst und Kunstgewerbe. - Das deutsche Heer. - Die deutsche Industrie. - Mit drei Einschaltbildern und dreiundzwanzig Textabbildungen in Tondruck nach Photographien.

14.2 (1899/1900) 577-592 - Hanns von Zobeltiß: Von der Weltausstellung zu Paris. III. Mit Zwei Einschaltbildern und zwanzig Textillustrationen in Tondruck und Photographien. 


\section{Die Gartenlaube}

48 (1900) 480-483, 512-516, 544-548, 640-643, 701-706 und 768-770 - J. C. Heer: Spaziergänge durch die Weltausstellung in Paris.

48 (1900) 352-354 - Felix Vogt: Deutsche Bauten auf der Pariser Weltausstellung.

\section{Andere}

Revue des deux mondes 132 (1895) 888-908 - Octave Mirbeau: Pourquoi des Expositions?

Illustrierte Zeitung 51 (1868) 3 f. - Die Illustration als Hebel der Volksbildung. 
Abbildungen

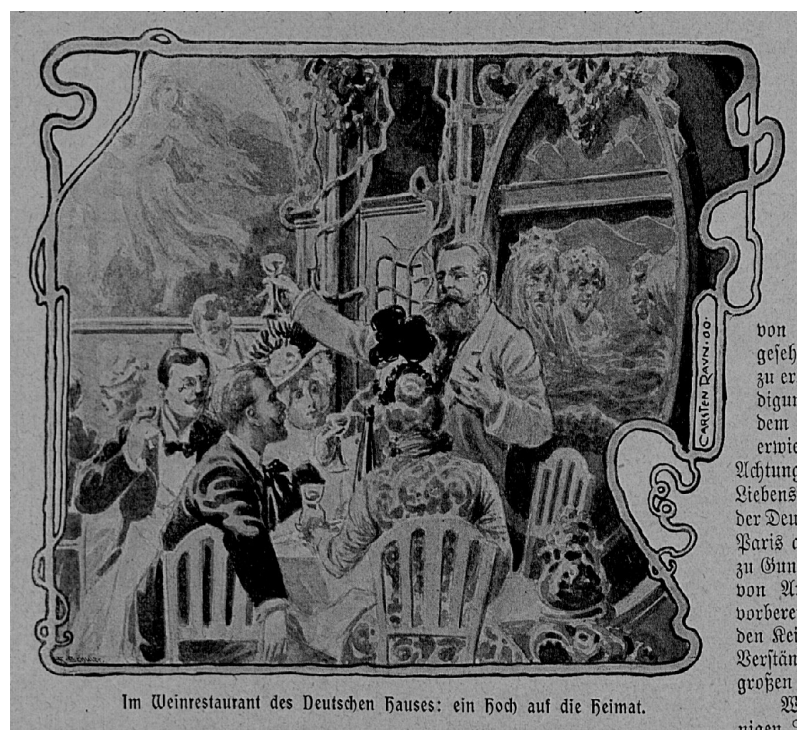

Illustration 1

(Die Gartenlaube, Band 48, S. 547).

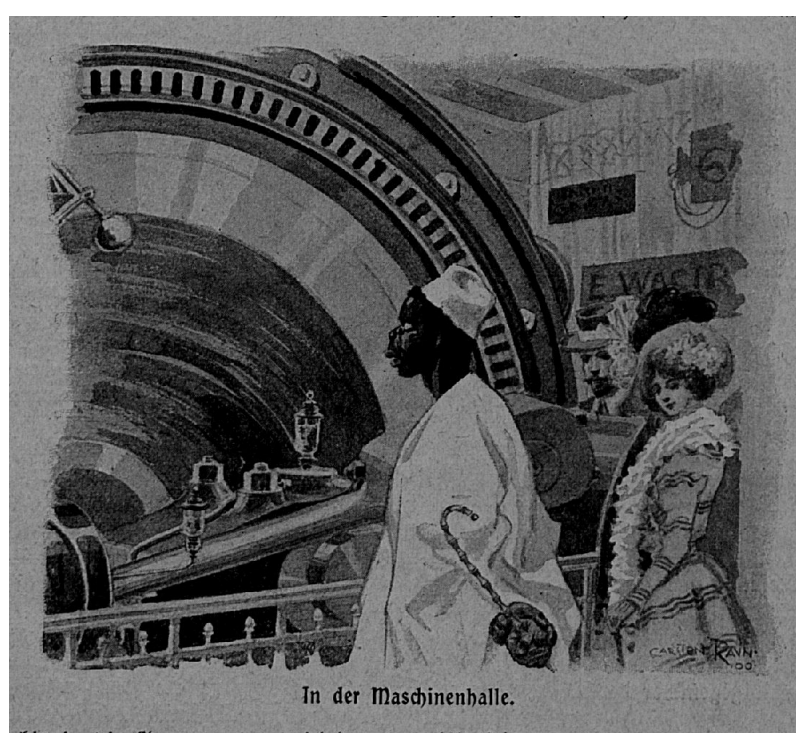

Illustration 2

(Die Gartenlaube, Band 48, S. 624). 


\section{Literaturverzeichnis}

Barth, Dieter: Zeitschrift für Alle. Das Familienblatt im 19. Jahrhundert. Ein sozialhistorischer Beitrag zur Massenpresse in Deutschland, Münster (Univ.) 1974, 203-205.

Benjamin, Walter: Paris, die Hauptstadt des XIX. Jahrhunderts, in: ders.: Schriften, hg. von Theodor W. Adorno und Gretel Adorno, Bd. 1, Frankfurt am Main (Suhrkamp) 1955, 406-422.

Butzer, Günter/Günter, Manuela: Literaturzeitschriften der Jahrhundertwende, in: Naturalismus, Fin de siècle, Expressionismus 1890-1918, hg. von York-Gothart Mix, München (Hanser) 2000, 116-136.

Florack, Ruth: Tiefsinnige Deutsche, frivole Franzosen. Nationale Stereotype in deutscher und französischer Literatur, Stuttgart [u. a.] (Metzler) 2001.

Gaillard, Marc: Paris. Les Expositions universelles de 1855 à 1937, Paris (Presses Franciliennes) 2003, 74-142.

Gall, Lothar: Krupp. Der Aufstieg eines Industrieimperiums, Berlin (Siedler) 2000.

Graf, Andreas: Familien- und Unterhaltungszeitschriften, in: Geschichte des deutschen Buchhandels im 19. und 20. Jahrhundert. Band 1: Das Kaiserreich 1871-1918. Teil 2, hg. von Georg Jäger, Frankfurt am Main (MVB) 2003, 409-522.

Haltern, Utz: Die „Welt als Schaustellung”. Zur Funktion und Bedeutung der internationalen Industrieausstellung im 19. und 20. Jahrhundert, in: Vierteljahrschrift für Sozial- und Wirtschaftsgeschichte 60 (1973) 1-40.

Hofmann, Werner: Die Welt als Schaustellung, in: ders.: Das irdische Paradies. Kunst im neunzehnten Jahrhundert, München (Prestel) 1960, 151-181.

Kirschstein, Eva-Annemarie: Die Familienzeitschrift. Ihre Entwicklung und Bedeutung für die deutsche Presse, Charlottenburg (Lorentz) 1937.

Koch, Marcus: Nationale Identität im Prozess nationalstaatlicher Orientierung. Dargestellt am Beispiel Deutschlands durch die Analyse der Familienzeitschrift ,Die Gartenlaube' von 1853-1890, Frankfurt am Main [u. a.] (Peter Lang) 2003.

Krutisch, Petra: Aus aller Herren Länder. Weltausstellungen seit 1851, Nürnberg (Germanisches Nationalmuseum) 2001, 95-103.

Kuchenbuch, Thomas: Die Welt um 1900. Unterhaltungs- und Technikkultur, Stuttgart [u. a.] (Metzler) 1992.

Mandell, Richard Donald: Paris 1900 - The Great World's Fair, Toronto (University of Toronto Press) 1967.

Mitterand, Henri: Zola. Bd. 3: L'honneur (1893-1902), Paris (Fayard) 2002.

Morand, Paul: 1900, Paris (Éditions de France) 1931.

Naumann, Friedrich: Ausstellungsbriefe, Berlin-Schöneberg (Buchverlag der „Hilfe“) 1909.

Ory, Pascal: Les Expositions universelles de Paris, Paris (Ramsay) 1982.

Schneider, William H.: Colonies at the 1900 World Fair, in: History Today 31 (1981) H. 5, 31-36.

Syndram, Karl Ulrich: Rundschau-Zeitschriften. Anmerkungen zur ideengeschichtlichen Rolle eines Zeitschriftentyps, in: Ideengeschichte und Kunstwissenschaft im Kai- 
serreich, hg. von Ekkehard Mai, Stephan Waetzoldt und Gerd Wolandt, Berlin (Mann) 1983, 349-370.

Wehler, Hans-Ulrich: Nationalismus, München (Beck) 2004.

Wolbring, Barbara: Krupp und die Öffentlichkeit im 19. Jahrhundert. Selbstdarstellung, öffentliche Wahrnehmung und gesellschaftliche Kommunikation, München (Beck) 2000.

Wörner, Martin: Vergnügen und Belehrung. Volkskultur auf den Weltausstellungen 18511900, Münster [u. a.] (Waxmann) 1999.

Zola, Émile: La Débâcle, hg. von Henri Mitterand, Paris (Gallimard) 1984. 